\title{
Effects of Laser Peening Parameters on Plastic Deformation in Aqueous Glycerol Solution as Plasma Confinement Layer
}

\author{
Noor Shahira Masroon ${ }^{* 1}$, Hikaru Hirata ${ }^{1}$, Miho Tsuyama $^{1}$, Manabu Heya², and Hitoshi Nakano ${ }^{1}$ \\ ${ }^{1}$ Faculty of Science and Engineering, Kindai University, Japan \\ ${ }^{2}$ Faculty of Science and Engineering, Osaka-Sangyo University, Japan \\ *Corresponding author's e-mail: shahiramasroon@kindai.ac.jp
}

\begin{abstract}
Laser peening is a surface treatment technique that can improve the properties of metallic materials. Installation of the plasma confinement layer with liquid plays an important role to obtain high magnitude shock wave pressure. In this study, the effects of laser peening parameters in glycerol solution as plasma confinement layer were investigated through hardness and residual stress measurements. The stainless steel, SUS316L, has been used as a target sample. The experiments were carried out by varying the concentration of glycerol solution. Laser peening treatment under a liquid confining medium is advantageous because a dual-impulse peening effect can be obtained by the shock wave pressure due to the confinement of laser-induced plasma and collapse of the laser-induced cavitation bubble. The impact from both shock wave pressures and dynamics of a laser-induced cavitation bubble have been evaluated to consider deeply the effects of laser peening parameters on the hardness and residual stress of laser-peened samples. Glycerol solution around $20 \mathrm{wt} \%$ in concentration was found to be desirable in any laser peening parameters to achieve efficient laser peening treatment.
\end{abstract}

DOI: $10.2961 /$ jlmn.2021.03.2002

Keywords: laser peening, cavitation bubble, plasma confinement layer, acoustic impedance, shock wave pressure

\section{Introduction}

The laser peening process under a confinement medium has shown great potential in many areas, such as the maintenance and repair of nuclear power plants and bridges [1]. A high magnitude shock wave pressure can be induced into the metal efficiently, improving the material's strength, corrosion resistance, and fatigue performance. Laser peening generates tailored compressive residual stress fields that can be easily monitored and controlled by selecting laser peening parameters [2].

The installation of the confining medium plays an important role in the laser peening process. The confining medium is also called as "plasma confinement layer" because it confines the expansion of laser-induced plasma from rapidly expanding away from the metal surface, thus contributes to an increase in the magnitude of the shock wave pressure up to several GPa $[3,4]$. The peak pressure owing to the laser plasma from the laser irradiation in confining medium can be estimated by

$$
\begin{aligned}
& P=0.01 \sqrt{\frac{\alpha}{2 \alpha+3}} \sqrt{Z \cdot I_{0}} \\
& \frac{2}{Z}=\frac{1}{Z_{T}}+\frac{1}{Z_{C}}
\end{aligned}
$$

where $P, Z$, and $I_{0}$ are the peak pressure, acoustic impedance, and laser intensity, respectively [3]. $\alpha$ is a constant that corresponds to the ratio of thermal to internal energy, and it is shown to be $0.3-0.5$, depending on the laser parameters. In Eq. (2), $Z$ is defined as the reduced acoustic impedance, where $Z_{T}$ and $Z_{C}$ are the acoustic impedances of the target sample and confining medium, respectively. The degree of confining medium was determined by acoustic impedance. The acoustic impedance $Z_{C}$ is given by the product of the density $d_{C}$ and the speed of sound $v_{S}$ in the medium.

$$
Z_{C}=d_{C} \times v_{S}
$$

The confinement can be any medium that has characteristics of high transmissivity at the laser wavelength, no chemical reactivity with the target materials, and non-flammability.

In the case of laser peening treatment under a liquid confining medium, there are two mechanisms involved to generate shock wave: laser-induced plasma and laser-induced cavitation bubble. Recently, the phenomena of laser-induced cavitation bubble on a solid target is considered to be important due to the cavitation impact that can enhance the performance of metallic properties and it has gradually attracted many researchers $[5,6]$. Several studies have shown that laser peening treatment in a liquid confining medium is advantageous because a dual-impulse peening effect can be obtained by a single-shot laser pulse $[7,8]$. When a short-pulsed laser is focused on a metal that is immersed in a confining medium, as described above, a high-pressure laser-induced plasma is generated on the metal's surface. The plasma confinement layer confines the plasma from rapidly expanding away, thus enhances a shock wave that propagates into the 
metal. Subsequently, a shock wave propels the surrounding liquid and resulting in a pressure drop. Due to the pressure drop induction, a laser-induced cavitation bubble is generated on the metal surface due to the motion of the surrounding liquid. This cavitation bubble expands to its maximum size, collapses, and rebounds many times in a confining medium. The collapse of the cavitation bubble emits a strong shock wave on the metal surface. Plastic deformation occurs due to the shock wave pressure that propagates through the metal and exceeds the yield strength of the material; subsequently, work-hardening, and compressive residual stress are induced on the metal surface. Hence, the effect of laser peening can be expressed as bellow.

$$
E_{L P} \propto \int_{0}^{\infty} P_{(t)} d t
$$

In Eq. $4, E_{L P}$ is the effect of laser peening and $P_{(t)}$ is the shock wave pressure in time dependent. Here, the effect of laser peening in a plasma confinement layer is attributed to the impact of shock waves generated from the confinement of laser-induced plasma $P_{L}$ and the collapse of the cavitation bubble $P_{C}$ in hundreds of microseconds later from laser irradiation. Therefore, both $P_{L}$ and $P_{C}$ are important factors in determining the effectiveness of laser peening treatment.

In most studies involving the liquid confinement in laser peening treatment, water has been most often used because it is convenient to use. Recently, several studies have already demonstrated that glycerol solution is effective as an alternative for substituting water as a plasma confinement layer because it can enhance the shock wave pressure in laser-induced shock processing due to higher acoustic impedance [9-11]. The acoustic impedance of glycerol solution can be increased easily by increasing its concentration. However, Tsuyama et al. reported that the high concentration of glycerol solution causes a decrease in the laser peening efficiency due to the bubble formation in high-viscous solution [11]. To the best of our knowledge, the systematic experimental studies regarding the desirable laser peening parameters in glycerol solution does not consider properly the behavior of laser-induced cavitation bubble and its effect on the laser peening efficiency. Therefore, first, this study aims to achieve effective laser peening treatment by investigating the effect of laser peening parameters on the plastic deformation of stainless steel in an aqueous glycerol solution. The experiments were carried out by varying the concentration of aqueous glycerol solution, coverage, and laser intensity. Subsequently, the effects of concentration of aqueous glycerol solution on the shock wave pressure and the dynamics of the cavitation bubble were investigated to gain a deeper understanding of the phenomena occurring in the aqueous glycerol solution near the surface of the target sample during laser peening treatment.

\section{Method}

\subsection{Laser peening treatment}

SUS316L with dimensions of $25 \mathrm{~mm} \times 25 \mathrm{~mm} \times 5 \mathrm{~mm}$ was used as the target sample. The initial properties of the samples were standardized by performing an annealing treatment. During the annealing treatment, the samples were heated under vacuum at $900{ }^{\circ} \mathrm{C}$ for $3 \mathrm{~h}$.
The schematic configuration of the experimental setup for laser peening is shown in Fig. 1. A laser peening technique without a protective coating layer to impart compressive residual stress on the samples was adopted in all experiments [12]. The experiments were performed using secondharmonic radiation from a Q-switched Nd: YAG laser operating at $10 \mathrm{~Hz}$, a wavelength of $532 \mathrm{~nm}$, and a pulse width of 4 ns. The laser beam was passed through an energy attenuator and pinhole and then focused on the sample using a lens with a focal length of $10 \mathrm{~cm}$. The focal spot diameter was fixed at $200 \mu \mathrm{m}$ by adjusting the size of the pinhole. The sample was supported by a holder and placed in a transparent container, or "peening cell," which was filled with confinement media. The thickness of the confinement medium above the sample was $20 \mathrm{~mm}$, which is sufficient to suppress the expansion of the laser-produced plasma to generate high pressure. The peening cell was then clamped down on an XY stage. The laser beam passed through the confinement medium and struck the sample perpendicularly.

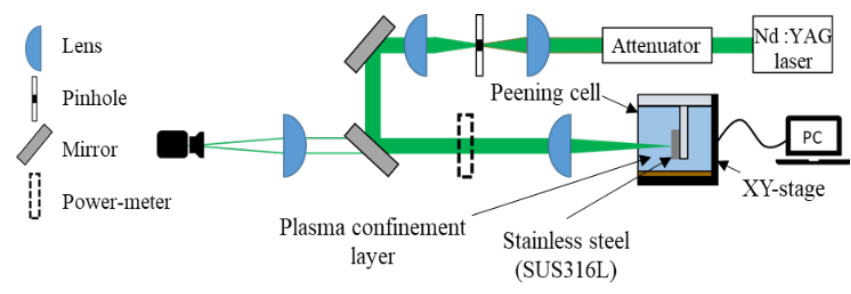

Fig. 1 Experimental setup for laser peening

Glycerol was used as the plasma-confinement layer. Various concentrations were obtained by mixing the glycerol solution with pure water. The peening coverage $C_{V}$, which is the number of laser shots per unit area, was obtained by controlling the XY-stage with a computer to move the target sample in the $\mathrm{x}$ and $\mathrm{y}$ directions during laser irradiation. Here, $C_{V}=\left(\pi d^{2} / 4\right) \cdot N / A \times 100[\%]$, where $d, N$, and $A$ represent the spot diameter of the irradiated laser pulse, number of pulses, and irradiated area, respectively. The laser peening effect was evaluated by measuring the work-hardening and residual stress at the surface of the metal. Work hardening was measured using a Vickers microhardness tester with a load of $1.961 \mathrm{~N}$ applied for $10 \mathrm{~s}$. The residual stress was measured using the X-ray diffraction method.

A high-speed video camera was used as the image recording device to observe the phenomena occurring in the aqueous glycerol solution near the surface of the target sample during laser peening treatment. The exposure time is set to be $8 \mu$ s and up to 100 consecutive images can be recorded in a single-shot laser pulse. The dynamics of a cavitation bubble generated in various concentrations of aqueous glycerol solutions after laser irradiation were examined.

\subsection{Measurement of shock wave pressure amplitude}

Shock wave pressure seems to be the most important measurable feature in laser peening [13]. The experimental arrangement to investigate the shock wave pressure generated from both the confinement of laser-induced plasma and the collapse of laser-induced cavitation bubbles is shown in Fig. 2. The experiments were performed with a Nd: YAG laser operating at a wavelength of $532 \mathrm{~nm}$, and a similar optical arrangement as the laser peening treatment was applied. The laser intensity was fixed at $2 \mathrm{GW} / \mathrm{cm}^{2}$. A SUS316L 
stainless steel sheet with a thickness of $0.05 \mathrm{~mm}$ was used as a target sample and glued on a polyvenyliden fluoride (PVDF) gauge sensor. The gauge sensor was connected to an oscilloscope via charge integrator (CI-50-0.1 Dynasen Inc.). The magnitude of shock wave pressure was evaluated by measuring the shock impulse of laser-induced plasma and the collapse of cavitation bubbles generated by a single-shot pulse laser in aqueous glycerol solutions of various concentrations. Figure 3 shows an example of the output signal from the PVDF gauge sensor produced by the confinement of laser-induced plasma $P_{L}$ and the collapse of cavitation bubble $P_{C}$. The impact of both shock impulses causes laser peening. By using Eq. (4), the shock impulse for both the laser-induced plasma and the collapse of cavitation bubble can be estimated simply by measuring the area under the curve of the peak pulse.

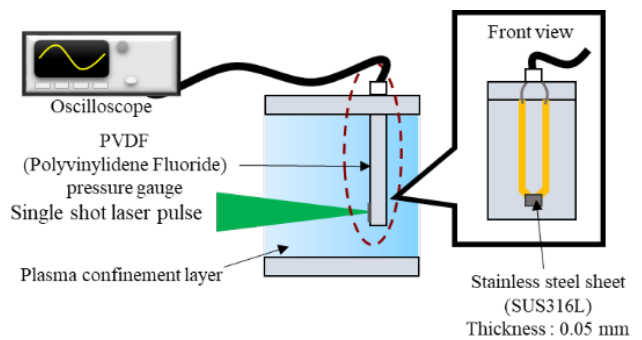

Fig. 2 Experimental setup for shock impulse measurement

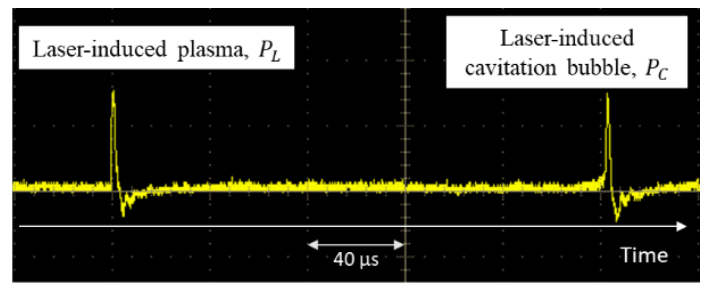

Fig. 3 Shock wave signal from laser-induced plasma and the collapse of laser-induced cavitation bubble from PVDF gauge sensor

\section{Results}

Figure 4 shows the hardness difference of the metal surface as a function of concentration at different coverages. The hardness difference is the increase in the surface hardness in the laser-peened area from its initial hardness. In water as the plasma confinement layer, the hardness obtained was plotted at a concentration of $0 \mathrm{wt} \%$. Throughout this work, the highest value of $98 \mathrm{HV}$ was obtained at $900 \%$ coverage when the concentration of the aqueous glycerol solution was approximately $20 \mathrm{wt} \%$. The metal hardness increases when the concentration is increased up to $20 \mathrm{wt} \%$ of concentration for all coverages. However, when the concentration is beyond $20 \mathrm{wt} \%$, the hardness decreased. In addition, according to the results shown in Fig 4, by increasing the coverage from $100 \%$ to $900 \%$, the metal hardness can be increased at any concentration. This shows that the coverage does not strongly related to the concentration of the aqueous glycerol solution.

The images of the laser-peened surface in the case of aqueous glycerol solution at $20 \mathrm{wt} \%$ and $80 \mathrm{wt} \%$ in concentration when the coverage and laser intensity were fixed at $900 \%$ and $2 \mathrm{GW} / \mathrm{cm}^{2}$, respectively, are shown in Fig. 5. A uniform overlapping dents pattern on the peened surface was obtained at a $20 \mathrm{wt} \%$ concentration. However, at $80 \mathrm{wt} \%$ concentration, an uneven overlapping dents pattern was obtained on the peened surface. This demonstrates that the laser beam cannot reach the metal surface successfully, resulting in decreased metal hardness. The concentration of the solution affected the laser-peening result. Therefore, this is considered in the discussion section.

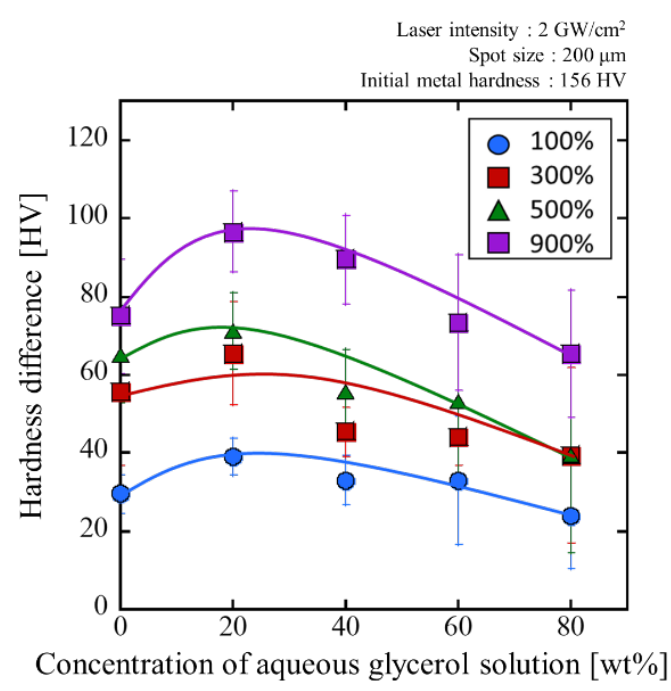

Fig. 4 Hardness difference as a function of the concentration of aqueous glycerol solution at different coverage

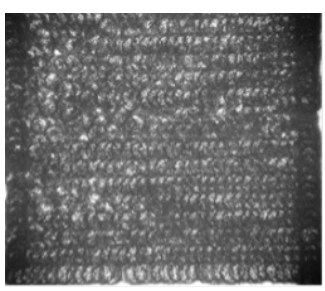

(a)

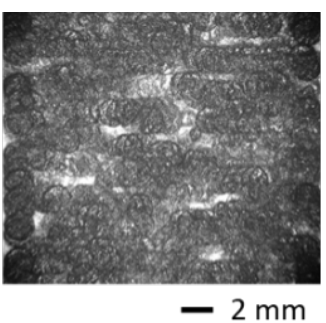

(b)
Fig. 5 Laser-peened surface of stainless steel when the concentration of aqueous glycerol solution is (a) $20 \mathrm{wt} \%$ and (b) $80 \mathrm{wt} \%$

An aqueous glycerol solution of around $20 \mathrm{wt} \%$ in concentration was desirable for obtaining high metal hardness. The effect of the laser intensity on the metal hardness was investigated using a $20 \mathrm{wt} \%$ aqueous glycerol solution. The hardness difference as a function of laser intensity is shown in Fig. 6. Based on Eq. (1), the peak pressure is proportional to the laser intensity. The results obtained in this study demonstrate that the metal hardness is almost proportional to the laser intensity up to $4 \mathrm{GW} / \mathrm{cm}^{2}$. However, the linear relationship between the laser intensity and metal hardness does not exist beyond a laser intensity of approximately $4 \mathrm{GW} / \mathrm{cm}^{2}$.

The compressive residual stress at the metal surface is beneficial to improve fatigue strength. To evaluate the induced residual stress, the target sample was characterized using the X-ray diffraction method. As shown in Fig. 7, the compressive residual stress was introduced by laser peening in two types of plasma confinement layers: water and aqueous glycerol solutions with a $20 \mathrm{wt} \%$ concentration. Although tensile stress was detected on the surface of the target 
metal (depth $=0 \mu \mathrm{m})$, the residual stress changed from tensile to compressive residual stress when the depth was increased in both the plasma confinement layers. The highest value of approximately $184 \mathrm{MPa}$ at a depth of approximately 10-40 $\mu \mathrm{m}$ under the metal surface was obtained in $20 \mathrm{wt} \%$ aqueous glycerol solution owing to the high acoustic impedance.

The shock wave pressure amplitude was investigated using a PVDF gauge sensor. The shock wave pressure amplitude delivered to the target can be determined by measuring both the shock impulses, $P_{L}$, and $P_{C}$ emitted by the laserinduced plasma and the collapse of the cavitation bubble. Figure 8 shows the total shock impulse as a function of the concentration of the aqueous glycerol solution. The total shock impulse, $P_{L}+P_{C}$, is defined as the total summation of both shock impulses mentioned above and indicated in Eq. (4). The total shock impulse increased when the concentration was increased. A peak at approximately $20 \mathrm{wt} \%$ concentration can be observed. The characteristic of the total shock impulse exhibits almost the same trend as that of the metal hardness.

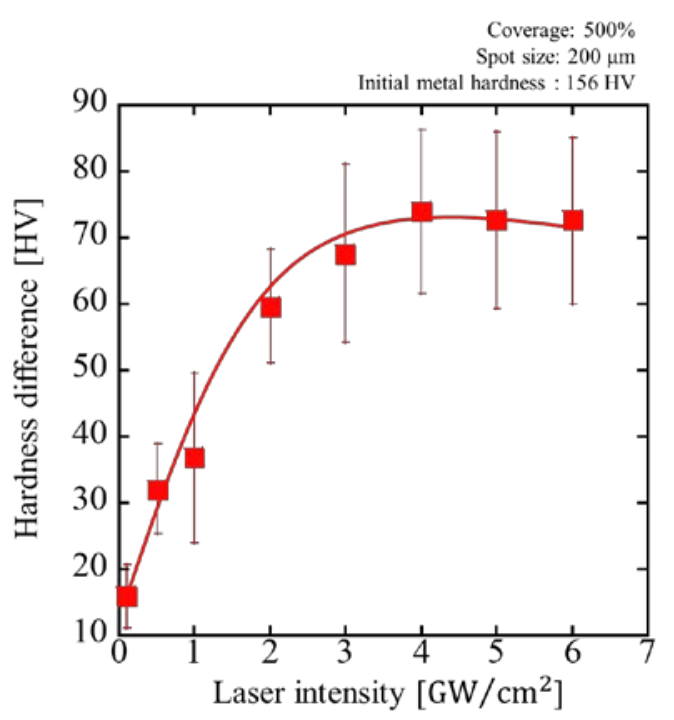

Fig. 6 Hardness difference as a function of laser intensity

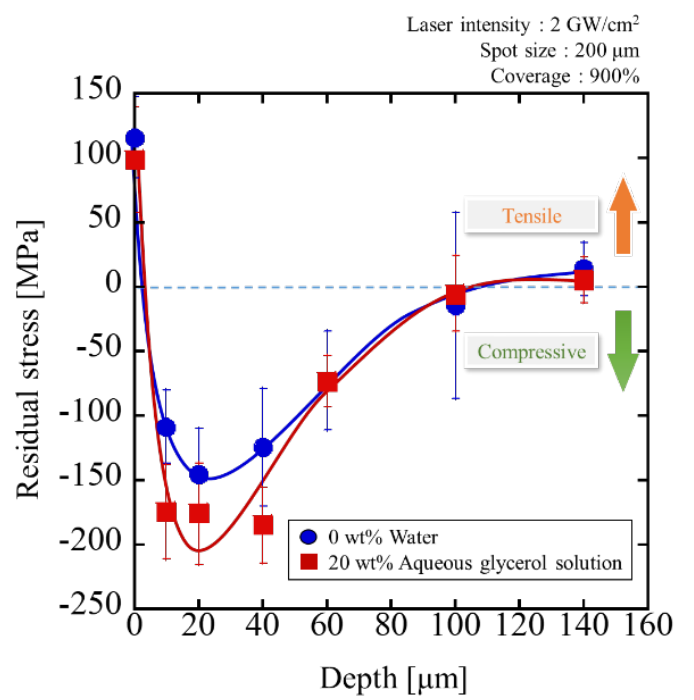

Fig. 7 Residual stress as a function of depth below the surface
To determine why laser peening effect decreased when the concentration was increased, the shock impulse from the collapse of first cavitation bubble was considered. Some researchers have reported that the collapse of first cavitation bubble is effective for underwater laser peening [5,14]. Figure 9 shows the shock impulse of the laser-induced cavitation bubble, that is, $P_{C}$, as a function of the concentration of the aqueous glycerol solution. The shock impulse by cavitation bubble showed the maximum value when the concentration was $20 \mathrm{wt} \%$. However, the shock impulse became weaker as the concentration of the aqueous glycerol solution increased from 20 to $80 \mathrm{wt} \%$. This shows that the shock impulse of the cavitation bubble is affected by the concentration of the plasma confinement layer; thus, a low concentration is desirable to obtain a high magnitude of shock impulse.

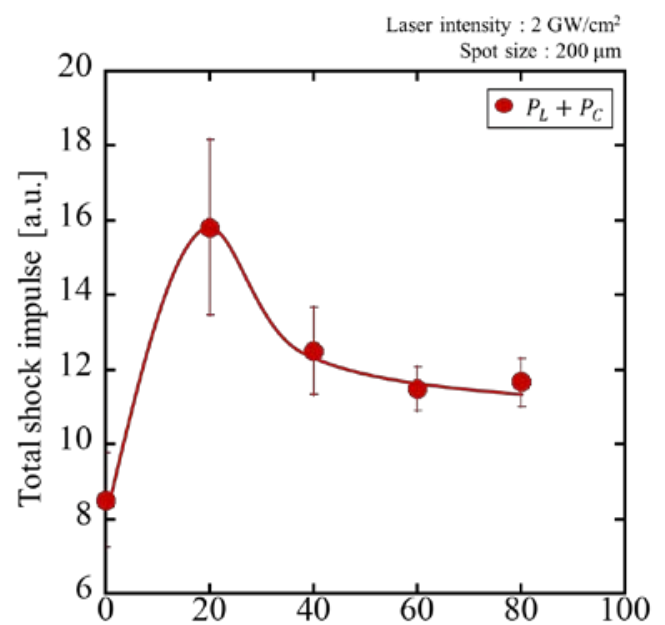

Concentration of aqueous glycerol solution [wt $\%$ ]

Fig. 8 Total shock impulse as a function of concentration of aqueous glycerol solution

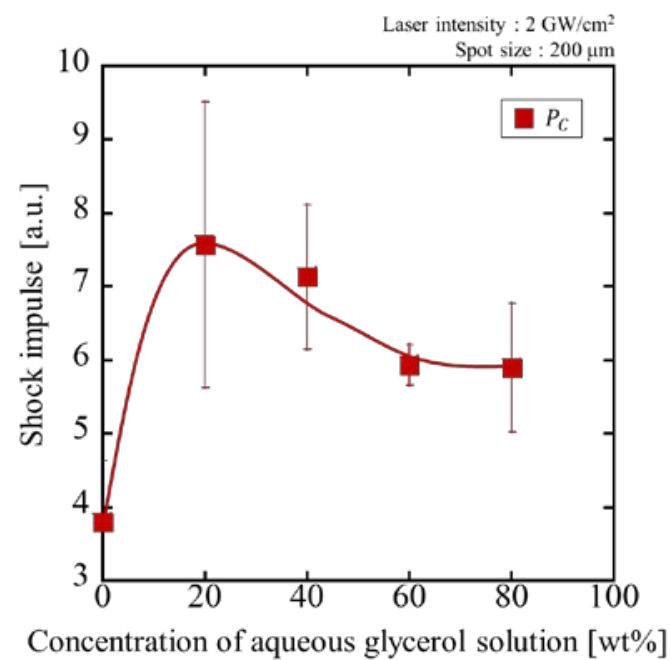

Fig. 9 Shock impulse of laser-induced cavitation bubble as a function of concentration of aqueous glycerol solution

\section{Discussion}

Our interest is to examine the desirable laser peening parameters for laser peening using a glycerol solution as a plasma confinement layer. According to the results obtained in Fig. 4, it was shown that the metal hardness can be improved when the concentration is around $20 \mathrm{wt} \%$. However, 
when the concentration is higher than $20 \mathrm{wt} \%$, the metal hardness decreases. This is most likely due to the concentration of glycerol solution which influences its viscosity. The lower value of metal hardness obtained in glycerol solution compared to water indicates that a high concentration is not effective for laser peening treatment. It should be noted that, after the first cavitation bubble collapsed, a new bubble was generated, expanded, shrank, and collapsed. This process continues until the bubble splits into small bubbles. Tsuyama et al. mentioned that laser peening treatment is not effective at high concentrations because of the small bubbles that remain on the optical path of the laser beam [11]. If the bubble remains in that position for more than $100 \mathrm{~ms}$ when the laser is operating at a frequency of $10 \mathrm{~Hz}$, the laser beam will be scattered and refracted [11].

In the low viscosity of the plasma confinement layer, the small bubble rises toward the surface of confining medium. However, in a highly viscous solution, the small bubble tends to stay along the laser incident axis for a long time because the rising speed becomes extremely slow. Consequently, the next laser pulse cannot reach the metal surface successfully because of the interruption of a small bubble along the laser incident axis. Therefore, an uneven overlapping dent pattern is obtained (Fig. 5(b)). Consideration of small bubbles is necessary when using a highly viscous medium as a plasma confinement layer in high-frequency laser processing.

In addition, we identified another reason for the decrease in metal hardness at high concentrations by measuring the laser-induced pressure and temporal evolution of bubble formation. The concentration of plasma confinement layer affects the shock impulse emitted from the collapse of the first cavitation bubble, as shown in Fig. 9. The shock impulse reaches a peak value of approximately $20 \mathrm{wt} \%$ aqueous glycerol solution. However, at a concentration of approximately $80 \mathrm{wt} \%$, a low-amplitude shock wave pressure is obtained. The dynamics of the laser-induced cavitation bubble up to hundreds of microseconds after laser irradiation on the target sample have been observed to explain that the increasing concentration of glycerol solution affects the magnitude of the shock impulse from the collapse of first cavitation bubble.

The evolution of the first laser-induced cavitation bubble in different concentrations of aqueous glycerol solution when focusing on $2 \mathrm{GW} / \mathrm{cm}^{2}$ of laser intensity on the target sample was captured by using a high-speed video camera with a time interval of $8 \mu \mathrm{s}$. The images were cropped to the desired field of view and are presented in Fig. 10. According to the temporal evolution of the cavitation bubble images shown in Fig. 10, after laser irradiation, a cavitation bubble with a hemispherical shape is generated and expands to its maximum size. Subsequently, the bubble becomes smaller and finally collapses. The largest cavitation bubble size was obtained at $20 \mathrm{wt} \%$ aqueous glycerol solution.

By observing the dynamics of laser-induced cavitation bubble using a high-speed video camera, we also found that the size of the first cavitation bubble qualitatively appears smaller when the concentration is increased. The maximum diameter of the first cavitation bubble at different concentrations of the aqueous glycerol solution was measured. The time-dependent changes in the generation of first cavitation bubble to the maximum bubble diameter after laser irradiation are shown in Fig. 11. The cavitation bubble reaches its maximum diameter of approximately $2.5 \mathrm{~mm}$ at $20 \mathrm{wt} \%$ concentration. A decrease in the diameter of the cavitation bubble can be observed when the concentration is increased owing to an increase in viscosity. In highly viscous solutions, viscous forces acting on the bubble resist bubble growth in the medium. Our study indicates that the decrease in the maximum diameter of the cavitation bubble results in a decrease in the shock wave pressure amplitude. This result demonstrates that the shock impulse emitted from the collapse of the first cavitation bubble is affected by the viscosity of the plasma confinement layer. Therefore, we conclude that the concentration of the aqueous glycerol solution was effective at around $20 \mathrm{wt} \%$.

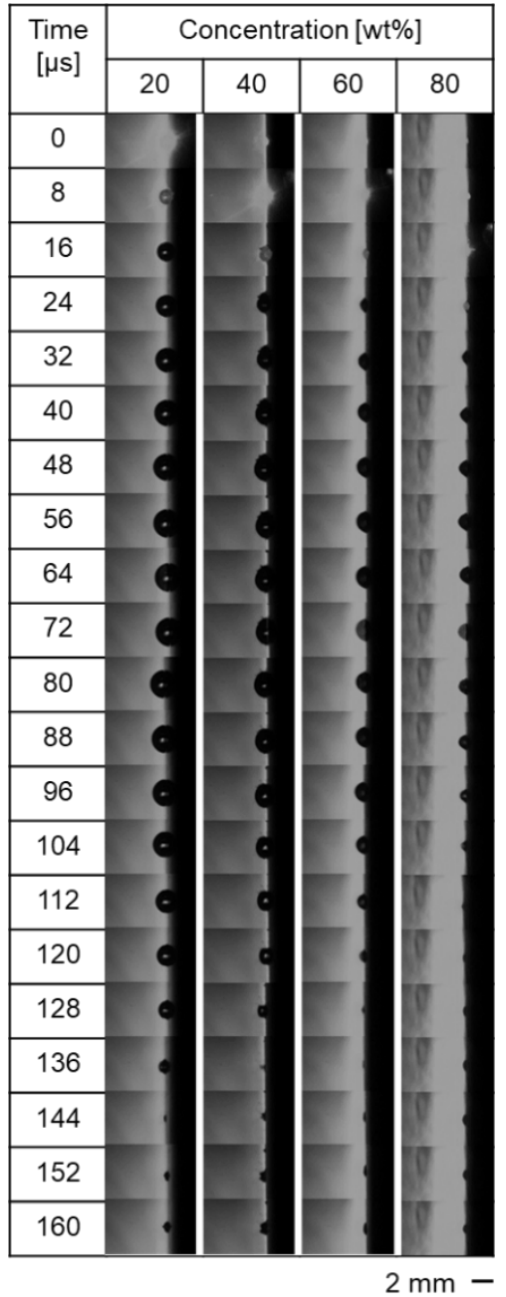

Fig. 10 Shadowgraph images after single laser pulse under different concentration of aqueous glycerol solution

Relatively high metal hardness can be obtained when the coverage is increased, as shown in Fig. 4. The metal hardness can be increased by applying a large number of laser pulses to the surface. At high concentrations, laser peening is not effective because of the low magnitude of the shock impulse emitted from the first cavitation bubble and the presence of a small bubble that interrupts the laser beam due to high viscosity. However, metal hardness can be improved by increasing the coverage. Despite that the coverage was only increased to $900 \%$ in this study, we believe that the 
coverage can be further increased to achieve a higher magnitude of metal hardness. Hence, a high coverage for laser irradiation is desirable for producing plastic deformation.

In the case of laser intensity dependence, the metal hardness can be increased up to $4 \mathrm{GW} / \mathrm{cm}^{2}$, as shown in Fig. 6 . The metal hardness increases owing to work hardening caused by the high shock wave pressure impact-induced inside the metal surface. When the laser intensity exceeds $4 \mathrm{GW} / \mathrm{cm}^{2}$, the metal hardness is no longer linearly proportional to the laser intensity. This is because laser-induced breakdown occurs, which limits the energy delivered to the metal surface [15]. Thus, a desirable laser intensity exists for effective laser peening treatment.

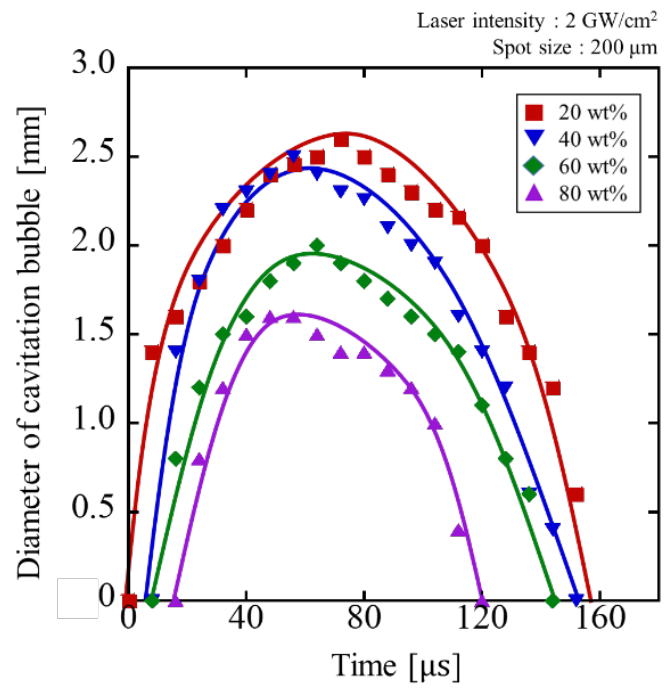

Fig. 11 Diameter of laser-induced cavitation bubble as a function of time

The compressive residual stress obtained using aqueous glycerol solution was higher than that of water because of the higher acoustic impedance. The high acoustic impedance of the plasma confinement layer contributes to an increase in the intensity of the shock wave pressure, thus enhancing the laser peening effect. A plasma confinement layer with high acoustic impedance is preferable for obtaining high residual stress.

The effectiveness of laser peening can be determined by the characteristics of the shock impulses of the laser-induced plasma and the collapse of laser-induced cavitation bubbles measured by a PVDF gauge sensor. The highest value of total shock impulse at $20 \mathrm{wt} \%$ of concentration shown in Fig. 8 corresponds to the results of metal hardness shown in Fig. 4. Furthermore, the large diameter of the first cavitation bubble, resulting in a high magnitude of shock impulse, contributes to the effectiveness of laser peening. This result demonstrates that an aqueous glycerol solution with a concentration of approximately $20 \mathrm{wt} \%$ can be employed as a plasma confinement layer to achieve a high degree of metal hardness and compressive residual stress.

\section{Conclusions}

The results of our study indicate that the effectiveness of laser peening treatment is affected by various factors. The effects of laser peening parameters on the plastic deformation of stainless steel in an aqueous glycerol solution were investigated, and efficient laser peening conditions were obtained. The highest value of $98 \mathrm{HV}$ was obtained at $900 \%$ coverage when the concentration of the aqueous glycerol solution was approximately $20 \mathrm{wt} \%$. An experimental study of the bubble dynamics in various concentrations of aqueous glycerol solution is useful in explaining how the viscosity of the solution affects the effectiveness of laser peening treatment. The results show that the acoustic impedance and viscosity of the plasma confinement layer must be balanced to achieve an effective laser peening treatment. The use of a glycerol solution as a plasma confinement layer can be of great advantage, especially in liquid-confined environment.

\section{References}

[1] J. Zhu, X. Jiao, C. Zhou, and H. Gao: Energy Procedia, 16 (2012) 153.

[2] A. Salimianrizi, E. Foroozmehr, M. Badrossamay, and H. Farrokhpour: Opt. Lasers Eng., 77 (2016) 112.

[3] R. Fabbro, J. Fournier, P. Ballard, D. Devaux, and J. Virmont: J. Appl. Phys., 68 (1990) 775.

[4] X. Hong, S. Wang, D. Guo, H. Wu, J. Wang, Y. Dai, X. Xia, and Y. Xie: Opt. Lasers Eng., 29 (1998) 447.

[5] D. Glaser and C. Polese: Appl. Phys. A, 123 (2017) 1.

[6] M. Senegačnik, K. Kunimoto, S. Yamaguchi, K. Kimura, T. Sakka, and P. Gregorčič: Ultrason. Sonochem., 73 (2021) 105460.

[7] H. Soyama: J. Mater. Process. Technol., 269 (2019) 65.

[8] H. Zhang, X. Ren, Y. Tong, E. Asuako Larson, S. AduGyamfi, J. Wang, and X. Li: Results Phys., 12 (2019) 1204.

[9] K. Elango, J.S. Hoppius, L.M. Kukreja, A. Ostendorf, and E.L. Gurevich: Surf. Coatings Technol., 397 (2020) 125988.

[10] T.T.P. Nguyen, R. Tanabe, and Y. Ito: Opt. Laser Technol., 100 (2018) 21.

[11] M. Tsuyama, N. Ehara, K. Yamashita, M. Heya, and H. Nakano: Appl. Phys. A, 124 (2018) 1.

[12] Y. Sano, K. Akita, K. Masaki, Y. Ochi, I. Altenberger, and B. Scholtes: J. Laser Micro Nanoeng., 1 (2006) 161

[13] L. Berthe, R. Fabbro, P. Peyre, L. Tollier, and E. Bartnicki: J. Appl. Phys., 82 (1997) 2826.

[14] T. Takata, M. Enoki, P. Chivavibul, A. Matsui, and Y. Kobayashi: Mater. Trans., 57 (2016) 1776.

[15] R. Fabbro, P. Peyre, L. Berthe, and X. Scherpereel: J. Laser Appl., 10 (1998) 265.

(Received: June 10, 2021, Accepted: November 23, 2021) 